Research Paper

\title{
The Role of Human Umbilical Vein Endothelial Cells in Osteogenic Differentiation of Dental Follicle-Derived Stem Cells in In Vitro Co-cultures
}

\author{
Jung-Suk Bok ${ }^{1}$, Sung-Hoon Byun ${ }^{1}$, Bong-Wook Park ${ }^{1}$, Young-Hoon Kang ${ }^{1}$, Sung-Lim Lee ${ }^{2}$, Gyu-Jin Rho ${ }^{2}$, \\ Sun-Chul Hwang ${ }^{3}$, Dong Kyun Woo ${ }^{4}$, Hyeon-Jeong Lee ${ }^{2,},{ }^{凶}$, June-Ho Byun ${ }^{1,}$ \\ 1. Department of Oral and Maxillofacial Surgery, Gyeongsang National University School of Medicine and Gyeongsang National University Hospital, \\ Institute of Health Sciences, Gyeongsang National University, Jinju, Republic of Korea \\ 2. Department of Theriogenology and Biotechnology, College of Veterinary Medicine, Gyeongsang National University, Jinju, Republic of Korea \\ 3. Department of Orthopaedic Surgery, Institute of Health Sciences, Gyeongsang National University School of Medicine, Jinju, Republic of Korea \\ 4. College of Pharmacy and Research Institute of Pharmaceutical Sciences, Gyeongsang National University, Jinju, Republic of Korea
}

$\square$ Corresponding authors: June-Ho Byun (Department of Oral and Maxillofacial Surgery, Gyeongsang National University School of Medicine and Gyeongsang National University Hospital, Institute of Health Sciences, Gyeongsang National University, Chilam-dong, Jinju, Republic of Korea, Tel: 82-55-750-8258, Fax: 82-55-761-7024, E-mail address: surbyun@gnu.ac.kr) or Hyeon-Jeong Lee (Department of Theriogenology and Biotechnology, College of Veterinary Medicine, Gyeongsang National University, Jinju, Republic of Korea, Tel: 82-55-772-2347, E-mail address: hjlee97@gnu.ac.kr ).

( ) Ivyspring International Publisher. This is an open access article distributed under the terms of the Creative Commons Attribution (CC BY-NC) license (https://creativecommons.org/licenses/by-nc/4.0/). See http://ivyspring.com/terms for full terms and conditions.

Received: 2018.05.17; Accepted: 2018.06.30; Published: 2018.07.30

\begin{abstract}
Angiogenesis and vascularization are essential for the growth and survival of most tissues. Engineered bone tissue requires an active blood vessel network for survival and integration with mature host tissue. Angiogenesis also has an effect on cell growth and differentiation in vitro. However, the effect of angiogenic factors on osteoprogenitor cell differentiation remains unclear. We studied the effects of human umbilical vein endothelial cells (HUVECs) on osteogenic differentiation of dental follicle-derived stem cells (DFSCs) in vitro by co-culturing DFSCs and HUVECs. Cell viability, based on metabolic activity and DNA content, was highest for co-cultures with a DFSC/HUVEC ratio of 50:50 in a 1:1 mixture of mesenchymal stem cell growth medium and endothelial cell growth medium. Osteoblastic and angiogenic phenotypes were enhanced in co-cultures with a DFSC/HUVEC ratio of 50:50 compared with DFSC monocultures. Increased expression of angiogenic phenotypes and vascular endothelial growth factor (VEGF) levels were observed over time in both 50:50 DFSC/HUVEC co-cultures and DFSC monocultures during culture period. Our results showed that increased angiogenic activity in DFSC/HUVEC co-cultures may stimulate osteoblast maturation of DFSCs. Therefore, the secretion of angiogenic factors from HUVECs may play a role in the osteogenic differentiation of DFSCs.
\end{abstract}

Key words: Dental follicle-derived stem cells; human umbilical vein endothelial cells; co-culture; osteogenic differentiation

\section{Introduction}

Bone is a dynamic tissue with a significant capacity to re-generate. One major reason for the self-healing ability of the bone is its high degree of vascularization. Vascularization is essential for bone remodeling during normal development, bone fracture repair, engraftment of autogenous bone, or bone tissue engineering in vivo. Vascular disruption due to bone damage leads to the formation of a hypoxic zone at the injury site. This hypoxic zone is believed to stimulate angiogenesis to restore blood flow to the damaged site. In vitro models of cellular and molecular interactions between blood vessels and bone cells also show that there are reciprocal functional interactions between endothelial cells or endothelial progenitor cells and osteoblast-like cells during osteogenesis [1-3].

Stem/progenitor cells have recently been developed as regenerative alternatives to autologous bone grafting for the treatment of various bone defects. The mesenchymal stem cells (MSCs) for cell-based or tissue-engineered regeneration can be isolated from small biopsy specimens obtained from outpatients under local anesthesia. These cells can be expanded in culture and can differentiate into distinct 
cell lineages under particular conditions. The major source of MSCs in adults is the bone marrow, where they exist in the supporting stroma. Bone marrow-derived MSCs are multipotent and are the designated gold standard for differentiating into osteogenic cells capable of forming bones in vitro. However, the process of isolating MSCs from bone marrows of patients can be complicated and painful [4-6].

The dental tissue provides an alternative source for osteogenic precursor cells. The tooth germ, also known as the tooth bud, is a primordial structure from which a tooth is formed. The tooth germ, which usually disappears upon tooth formation, exists in the slow-growing third molar of humans until the age of 10 to 16 years. The tooth germ consists of the enamel organ, the dental papilla, and the dental follicle. The dental follicle surrounds the tooth germ during the early stages of tooth development, and plays a crucial role in tooth eruption and development. Third molar tooth germs have been extracted from young adults for various reasons, including orthodontic treatments. The dental follicle from the extracted tooth germ is an alternative source of osteogenic cells for cell-based regenerative medicine. The dental follicle-derived stem cells (DFSCs) have been shown to differentiate into active osteoblasts that are involved in the mineralization of the bone matrix [7-11].

Bone regeneration can be enhanced by improving angiogenesis, a process regulated by various angiogenic factors. The combined delivery of osteogenic and angiogenic factors is more effective at promoting functional fracture healing compared with osteogenic factors alone, and is a novel approach in bone tissue engineering [12-16]. However, the mechanisms by which angiogenic factors impact osteoprogenitor cell differentiation are unknown. Enhanced angiogenesis does not always correlate with enhanced osteogenesis, and the effects of angiogenic factors on osteoprogenitor cell differentiation remain controversial. Some studies have shown no difference in the extent of angiogenesis in fracture nonunions and fracture unions [17-20].

Human umbilical vein endothelial cells (HUVECs), a prominent subset of macrovascular endothelial cells, are commonly used to evaluate the function and pathology of endothelial cells (e.g., angiogenesis). Several studies have reported the effects of angiogenic factors on osteo/odontogenic potential of dental stem cells [21-24]. However, the effects of the HUVECs on osteogenic differentiation of DFSCs are unknown. Therefore, we studied the role of HUVECs in osteogenic differentiation by co-culturing HUVECs with DFSCs.

\section{Materials and Methods}

\section{Culture of hDFSCs}

Dental follicles were harvested from lower impacted third molars that were surgically extracted from patients aged 13 to 15 years after obtaining their informed consent, as required by the Ethics Committee of Gyeongsang National University Hospital. The dental follicles were minced into 1-3 $\mathrm{mm}^{2}$ pieces, and cultured at $37^{\circ} \mathrm{C}$ in $95 \%$ humidified air and 5\% $\mathrm{CO}_{2}$, in 100-mm culture dishes containing Dulbecco's Modified Eagle's Medium (DMEM) supplemented with 10\% heat-inactivated fetal bovine serum (FBS), $100 \mathrm{IU} / \mathrm{mL}$ penicillin, and $100 \mu \mathrm{g} / \mathrm{mL}$ streptomycin. Upon reaching 90\% confluence, adherent cells were passaged by gentle trypsinization and reseeded in fresh medium. The medium was changed every 3 days during the induction period and cells at passages 3-5 were used.

\section{Analysis of surface markers}

Flow cytometry (FACSCalibur, Becton Dickinson, CA, USA) was used to detect surface antigens on DFSCs $\left(1 \times 10^{5}\right.$ cells per marker $)$. Specifically, cells were analyzed for the presence of mesenchyme markers (CD44, CD73, CD90, and CD105) and the absence of the hematopoietic markers CD34 and CD45, as described previously [25]. DFSCs that were at approximately $90 \%$ confluence were trypsinized, fixed in $3.7 \%$ formaldehyde solution, and incubated with fluorescein isothiocyanate (FITC)-conjugated primary antibodies (mouse anti-human) against CD34 (BD Pharmingen, CA, USA), CD44 (BD Pharmingen), CD45 (Santa Cruz Biotechnology, Santa Cruz, CA, USA), and CD90 (BD Pharmingen). For analysis of CD105 (mouse monoclonal, Santa Cruz Biotechnology) and CD73 (mouse monoclonal, BD Bioscience, CA, USA) expression, cells were treated with primary antibodies for 1 hour at $4^{\circ} \mathrm{C}$, washed with Dulbecco's phosphate buffered saline, and treated with FITC-conjugated goat anti-mouse immunoglobulin G (IgG) [BD Pharmingen] for 1 hour at $4^{\circ} \mathrm{C}$ in the dark. A total of 10,000 labeled cells were acquired and results were analyzed using BD FACSVerse ${ }^{\mathrm{TM}}$ with BD FACSuite $^{\mathrm{TM}}$ software.

\section{DFSCs differentiation into mesenchymal cell lineages in vitro}

DFSCs at passages 3-5 were evaluated for their ability to differentiate into adipogenic and osteogenic cell lineages. The cells were cultured in lineage-specific media for 21 days and the medium was changed every 3 days. Osteogenic induction medium was composed of DMEM, supplemented 
with $10 \%$ FBS, $50 \mu \mathrm{g} / \mathrm{mL}_{\mathrm{L}}$-ascorbic acid 2-phosphate, $10 \mathrm{nM}$ dexamethasone, and $10 \mathrm{mM}$ $\beta$-glycerophosphate. Osteogenesis was confirmed by alizarin red $S$ and von Kossa staining. Adipogenic medium consisted of $1 \mu \mathrm{M}$ dexamethasone, $10 \mu \mathrm{M}$ insulin, $100 \mu \mathrm{M}$ indomethacin, and $500 \mu \mathrm{M}$ isobutylmethylxanthine. For the detection of lipid droplets, differentiated cells were stained with oil red O solution for $30 \mathrm{~min}$.

\section{Culture of HUVECs and immunocytochemical analysis}

HUVECs were purchased from Gibco and cultured in Medium 200 (Gibco, ON, Canada) supplemented with low serum growth supplement (LSGS) (Gibco, ON, Canada) at $37^{\circ} \mathrm{C}, 95 \%$ humidified air, and $5 \% \mathrm{CO}_{2}$. The medium was changed every 3 days during the induction period and cells at passages 3-5 were used.

CD31 expression, which indicated the presence of endothelial cells, was visualized via immunocytochemical staining. The cells at passage 3 were rinsed with phosphate buffered saline (PBS), fixed with $4 \%$ paraformaldehyde for $20 \mathrm{~min}$, permeabilized with PBS containing 0.1\% Triton X-100 for 5 min, and blocked with PBS supplemented with $10 \%$ bovine serum albumin for 1 hour. The cells were first incubated with an antibody against CD31 (1:3200 dilution; Cell Signaling Technology, MA, USA) for 1 hour at room temperature. Cells were then incubated with FITC-conjugated donkey anti-mouse IgG secondary antibodies (1:100 dilution; Thermo Fisher Scientific, MA, USA) for 1 hour at room temperature. Cell nuclei were stained with $1 \mu \mathrm{g} / \mathrm{ml}$ 4',6-diamidino-2-phenylindole (DAPI), and the cells were observed using a fluorescence microscope and camera system (DM 4000B, Leica, Wetzlar, Germany).

\section{Co-culture of DFSCs with HUVECs}

DFSCs and HUVECs were co-cultured in 24-well plates at a density of $1 \times 10^{4}$ cells/well, at five different DFSC/HUVEC ratios (100:0, 75:25, 50:50, $25: 75$, and $0: 100$ ) in three different types of culture media: (i) MSC growth medium (MM) containing DMEM medium supplemented with $10 \%$ FBS, 100 $\mathrm{IU} / \mathrm{ml}$ penicillin, $100 \mu \mathrm{g} / \mathrm{mL}$ streptomycin and $2 \mathrm{mM}$ GlutaMAX ${ }^{\mathrm{TM}}-\mathrm{I}$, (ii) endothelial cell growth medium (EM) containing Medium 200 supplemented with LSGS, and (iii) 1:1 mixture of MM and EM. The medium was changed every $2-3$ days.

\section{Cell metabolic activity assay}

The metabolic activity of co-cultured cells with DFSCs and HUVECs was determined by the Alamar Blue-based metabolic assay (Invitrogen, CA, USA), according to the manufacturer's instructions. After 5 days of co-culturing DFSCs and HUVECs in different ratios and different types of media (as described above), $100 \mu \mathrm{l}$ of Alamar Blue reagent was added to each well at a final concentration of $10 \%(\mathrm{v} / \mathrm{v})$ and incubated for 4 hours at $37^{\circ} \mathrm{C}, 95 \%$ humidified air, and $5 \% \mathrm{CO}_{2}$. After incubation, $200 \mu \mathrm{l}$ of the supernatant from each well was transferred to a 96-well plate for optimal measurement by a microplate reader. The absorbance of each sample was measured at $570 \mathrm{~nm}$ by VersaMax ${ }^{\mathrm{TM}}$ Microplate Reader (Molecular Devices, CA, USA). The results of each experimental group were normalized to the values of the medium-only internal control group.

\section{DNA quantification assay}

Cellular DNA content was measured using QIAamp ${ }^{\circledR}$ DNA Mini Kit (Qiagen, CA, USA) according to the manufacturer's instructions. After 5 days of co-culturing DFSCs and HUVECs in different ratios and different types of media (as described above), the cells were trypsinized and the cell pellets were stored at $-80^{\circ} \mathrm{C}$ until DNA extraction. The samples were lysed with lysis buffer and proteinase K. DNA concentration and purity were measured with an OPTIZEN NANO $Q$ spectrophotometer (Mecasys, Daejeon, Korea), with an $\mathrm{A}_{260} / \mathrm{A}_{280}$ ratio of 2 \pm 0.2 , which indicated a pure preparation.

\section{Effects of HUVECs on osteoblastic phenotypes of DFSCs in vitro}

To examine the effects of HUVECs on osteoblastic phenotypes of DFSCs in vitro, DFSCs and HUVECs were co-cultured (DFSC/HUVEC ratio of 1:1) in 24-well plates at a density of $1 \times 10^{4}$ cells/well in a 1:1 mixture of $\mathrm{MM}$ and EM. At $60-70 \%$ confluence, the co-cultured cells were transferred to osteogenic induction medium and cultured for 21 days. As control, DFSCs were seeded in 24-well plates at a density of $1 \times 10^{4}$ cells/well in osteogenic induction medium for 21 days. The activity of alkaline phosphatase (ALP), an early marker for osteoblast differentiation, and mineralized nodule formation (indicative of maturation of the osteoblast phenotype) were used as indicators of osteoblast differentiation. ALP activity, alizarin red S and von Kossa staining, and calcium content were examined using a previously published method $[25,26]$.

\section{Quantitative PCR (qPCR) analysis}

The expression of the angiogenesis-related genes, vascular endothelial growth factor (VEGF) and angiotensin 1 (ANG1), was analyzed by qPCR in co-cultured cells (DFSC/HUVEC ratio of 1:1) during osteoblast differentiation in osteogenic induction medium at 7, 14, and 21 days of culture. Total RNA 
(500 ng) was used to synthesize complementary DNA (cDNA) using HiSenScript RH(-) RT PreMix kits (iNtRON Biotechnology, Seongnam, Korea). The cDNA reaction mixture $(20 \mu \mathrm{l})$ was incubated at $42^{\circ} \mathrm{C}$ for 1 hour. qPCR was performed using a Rotor-Gene $Q$ cycler (Qiagen, CA, USA) with 50 ng of cDNA and quantified with $2 \times$ Rotor-Gene SYBR Green Master Mix (Qiagen) using specific primer sets (Table 1). Reactions were performed with an initial denaturation at $95^{\circ} \mathrm{C}$ for $10 \mathrm{~min}$, followed by 40 cycles at $95^{\circ} \mathrm{C}$ for $10 \mathrm{~s}, 60^{\circ} \mathrm{C}$ for $6 \mathrm{~s}$, and $72^{\circ} \mathrm{C}$ for $6 \mathrm{~s}$. Rotor-Gene Q Series Software (Qiagen) was used to determine melting curves, amplification curves, and cycle threshold values. Gene expression levels were normalized to the corresponding control values of glyceraldehyde 3-phosphate dehydrogenase. All samples were run in triplicates and confirmed by $1.5 \%$ agarose gel electrophoresis.

Table 1. Primers used in $\mathrm{QPCR}$

\begin{tabular}{clll}
\hline $\begin{array}{c}\text { Target } \\
\text { gene }\end{array}$ & Sequence & $\begin{array}{l}\text { Product } \\
\text { size }(\mathrm{bp})\end{array}$ & $\begin{array}{l}\text { Annealing } \\
\text { temperature }\end{array}$ \\
\hline 1 VEGF & $\begin{array}{l}\text { F: AGAGACACATTGTTGGAA } \\
\text { R: GTTTCAATGGTGTGAGGACA }\end{array}$ & 125 & $60^{\circ} \mathrm{C}$ \\
2 ANG1 1 & $\begin{array}{l}\text { F: TCTTCTCTGCCTGTAAGTGTCC } \\
\text { R: GGTATTGCTACCTTGCCAACAA }\end{array}$ & $600^{\circ} \mathrm{C}$ \\
3 GAPDH & $\begin{array}{l}\text { F: AGTCAGCCGCATCTTCTTTT } \\
\text { R: CCAATACGACCAAATCCGTT }\end{array}$ & 189 & $60^{\circ} \mathrm{C}$ \\
\hline
\end{tabular}

\section{Enzyme-linked immunosorbent assay (ELISA) of VEGF}

In co-cultures of DFSCs and HUVECs that were cultured in osteogenic induction medium, culture media was collected after 48 houres at 7, 14, and 21 days of culture. VEGF levels were measured using the human VEGF ELISA Kit (Enzo Life Sciences, NY, USA). For each sample, $100 \mu \mathrm{l}$ of osteogenic-conditioned medium was assayed according to the manufacturer's instructions. Optical density was measured at a wavelength of $450 \mathrm{~nm}$ with a VersaMax ${ }^{\mathrm{TM}}$ Microplate Reader (Molecular Devices, CA, USA), and results were calculated using a calibration curve.

\section{Statistical analysis}

Each experiment was independently performed at least three times, and results from one experimental replicate for each case were shown as representative data. Data were expressed as mean \pm standard error of the mean and statistical analyses were computed using IBM SPSS 21.0 software. Data were evaluated using one-way analysis of variance (ANOVA), Tukey's multiple comparison test, and the Mann-Whitney test. Comparisons with $\mathrm{P}<0.05$ were considered statistically significant.

\section{Results}

\section{Characterization of DFSCS}

After passage 3, dental follicle-derived cells became relatively homogeneous and showed typical spindle-shaped morphology (Fig. 1A). Immunophenotypic analysis of cell surface antigens by flow cytometry showed that dental follicle-derived cells expressed the mesenchymal markers, CD44, CD73, CD90, and CD105, and were negative for the expression of hematopoietic stem cell markers, CD34 and CD45 (Fig. 1B). Cytochemical staining of the dental follicle-derived cells that were cultured in different induction media showed accumulation of lipid vacuoles and intracellular lipid droplets, and the deposition of mineralized extracellular matrix (Fig. 1C). This indicated that the dental follicle-derived cells successfully differentiated into mesenchymal cell lineages such as adipocytes and mature osteoblasts. Our results showed that the dental follicle-derived cells were phenotypically similar to MSCs, and were most likely DFSCs.

\section{CD31 expression in HUVECs}

HUVECs exhibited the characteristic rounded, cobblestone-like morphology at passage 3 (Fig. 2A), Immunocytochemical analysis showed that CD31, an endothelial marker, was expressed in HUVECs cultured in Medium 200 supplemented with LSGS (Fig. 2B).

\section{Optimization of DFSCs/HUVECs co-culture conditions}

To optimize conditions for co-cultures of DFSCs and HUVECs, co-cultures with varying DFSC/HUVEC ratios were grown in different culture media. Monocultures of DFSCs and HUVECs were used as controls. Cell metabolic activity and DNA content were used as output parameters to quantitatively assess cellular viability in these different culture conditions.

DFSCs cultured in MM showed increased cell metabolic activity compared with HUVECs. Higher cell metabolic activity was observed in DFSC/HUVEC co-cultures, regardless of co-culture ratios, compared with that in both DFSC and HUVEC monocultures. We observed no significant difference in cell metabolic activity between co-cultures with varying DFSC/HUVEC ratios. DFSCs cultured in EM also showed increased cell metabolic activity compared with HUVECs. We observed no significant difference in cell metabolic activity between DFSC/HUVEC co-cultures and DFSCs cultured in EM. Cell metabolic activity was the lowest in DFSC monocultures and highest in co-cultures with 
DFSC/HUVEC ratio of 50:50 grown in mixed culture media of MM and EM. A comparison of cells grown in different culture media revealed that co-cultures with a DFSC/HUVEC ratio of 50:50 grown in MM and EM mixed media showed the highest cell metabolic activity levels (Fig. 3A and B).

B
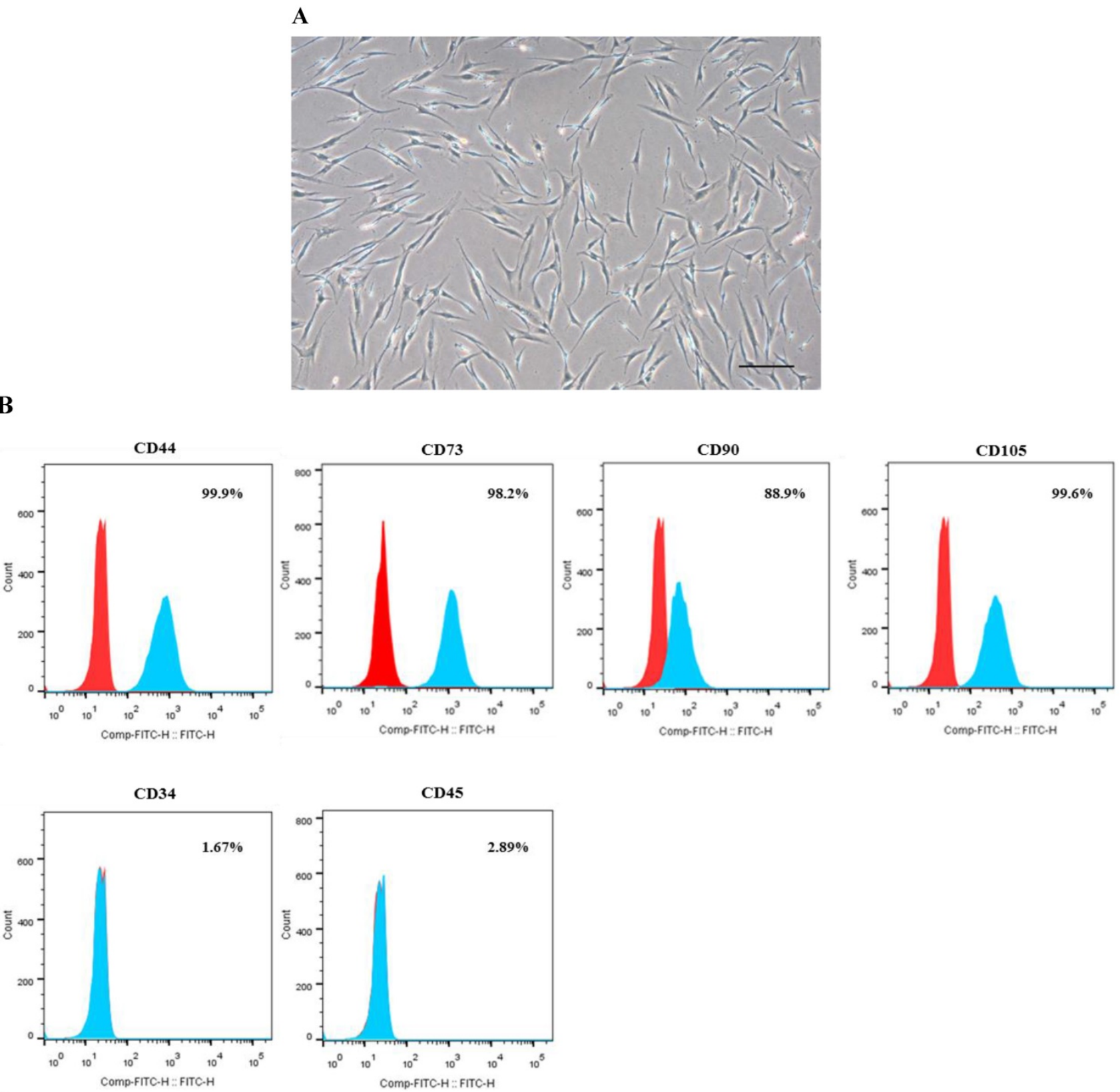

$\mathbf{C}$

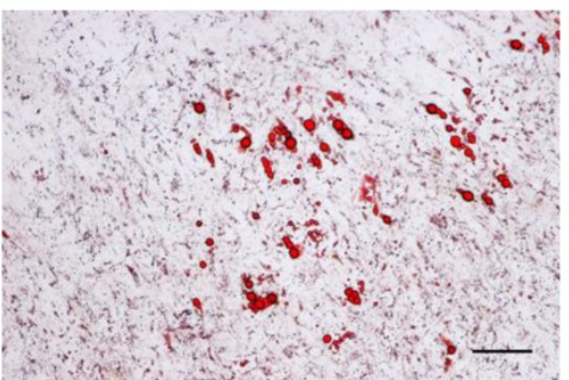

Oil red 0

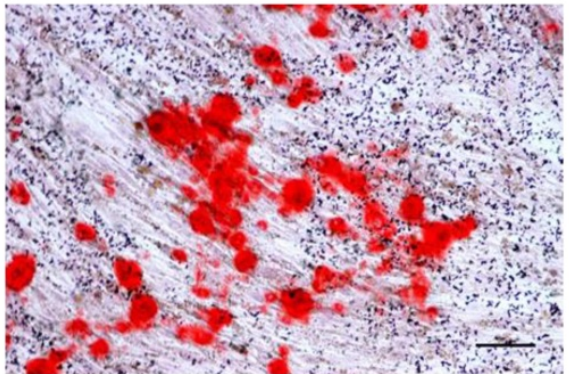

Alizarin red $\mathrm{S}$

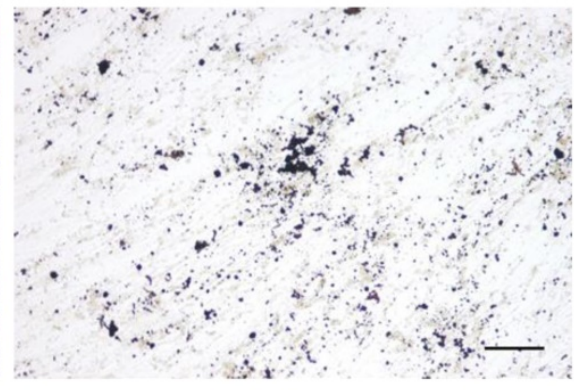

Von Kossa

Figure 1. Characterization of DFSCs. A: Differential interference contrast microscope image of DFSCs at passage 3 reveals a spindle-shaped morphology. Scale bar $=250 \mu \mathrm{m}$. B: DFSCs are positive for surface markers present on MSCs. C: Differentiation of DFSCs in vitro. Oil red O staining of lipid droplets indicated adipogenesis. Alizarin red S and von Kossa staining of mineralized nodules and calcium deposition indicated osteogenesis. Scale bar $=100 \mu \mathrm{m}$. 
A

B

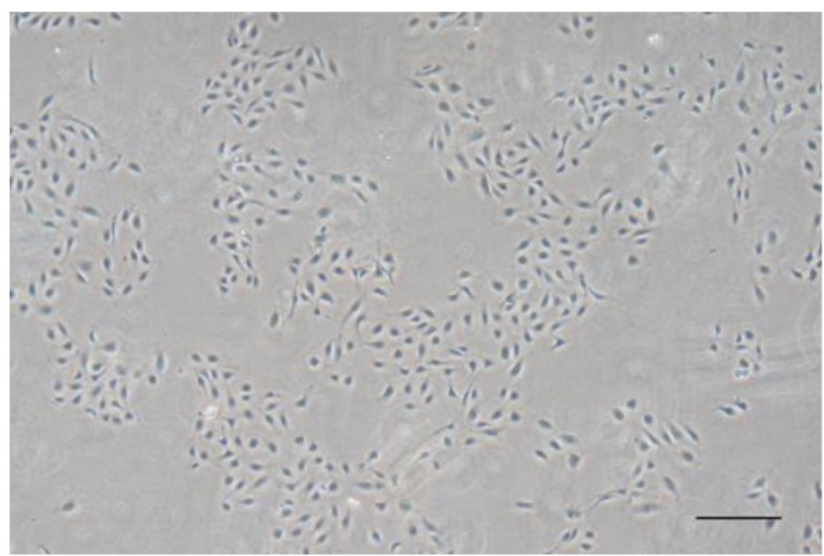

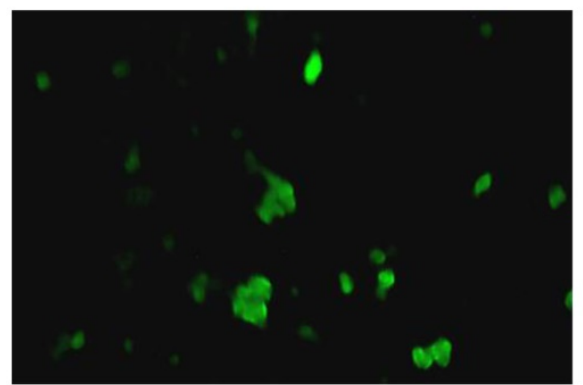

CD31

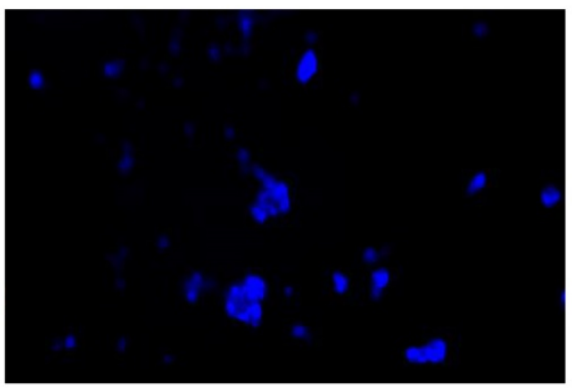

DAPI

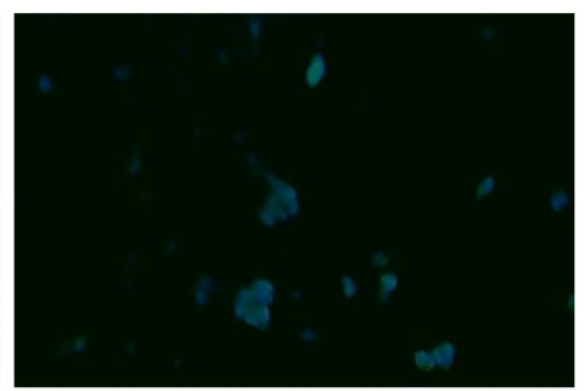

Combined

Figure 2. Culture of HUVECs. A: Rounded and cobblestone-like morphology of HUVECs at passage 3 . Scale bar $=500 \mu \mathrm{m}$. B: Fluorescent immunostaining for endothelial genetic marker CD31 expressed by HUVECs. 40× magnification.

HUVECs showed low levels of cellular DNA content regardless of culture medium. The DNA content of co-cultures and DFSC monocultures grown in MM was significantly higher than that of HUVEC monocultures. A comparison of co-cultures with varying DFSC/HUVEC ratios grown in MM showed low levels of DNA content in 25:75 co-cultures. DFSC monocultures, 75:25 co-cultures, and 50:50 co-cultures grown in MM showed similar levels of cellular DNA content. In cells grown in EM, cellular DNA content levels were the highest in DFSC monocultures and 50:50 co-cultures. DNA content levels were also the highest in DFSC monocultures and 50:50 co-cultures grown in MM and EM mixed media. A comparison of cells grown in different culture media revealed that co-cultures with a DFSC/HUVEC ratio of 50:50 grown in $\mathrm{MM}$ and $\mathrm{EM}$ mixed media showed the highest levels of DNA content (Fig. 3C and D). These results showed that optimal cell viability was achieved in co-cultures with a DFSC/HUVEC ratio of 50:50 grown in 1:1 mixed medium of MM and EM.

\section{Analysis of osteoblastic phenotypes in 50:50 co-culture}

Because the optimal co-culture ratio for DFSCs and HUVECs was shown to be 50:50 when cells were grown 1:1 MM and EM mixed media, we analyzed the expression of osteoblastic phenotypes in 50:50 co-cultures by measuring ALP activity, extent of mineralization, and calcium content levels in these cells. ALP activity was significantly higher in 50:50 co-cultures than in DFSC monocultures at 7 and 14 days of culture (Fig. 4A). DFSC/HUVEC 50:50 co-cultures also showed increased alizarin red- and von Kossa- positive mineralization, and calcium content levels when compared with DFSC monocultures at 14 and 21 days of culture (Fig. 4B and C). Our results suggest that osteogenic phenotypes of DFSCs could be enhanced via co-culture of DFSCs and HUVECs.

\section{Expression of angiogenesis-related genes in 50:50 co-cultures}

We studied the expression of angiogenesis-related genes, VEGF and ANG1, in 50:50 co-cultures of DFSCs and HUVECs. qPCR analysis showed that the expression of VEGF mRNA significantly increased at 7, 14, and 21 days of culture in 50:50 co-cultures compared with that in DFSC monocultures. DFSC/HUVEC 50:50 co-cultures showed the highest increase in ANG1 mRNA expression compared with DFSC monocultures at 14 days of culture (Fig 5A). 
A

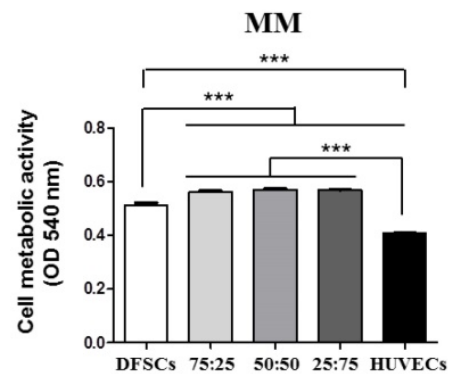

EM

MIX

B
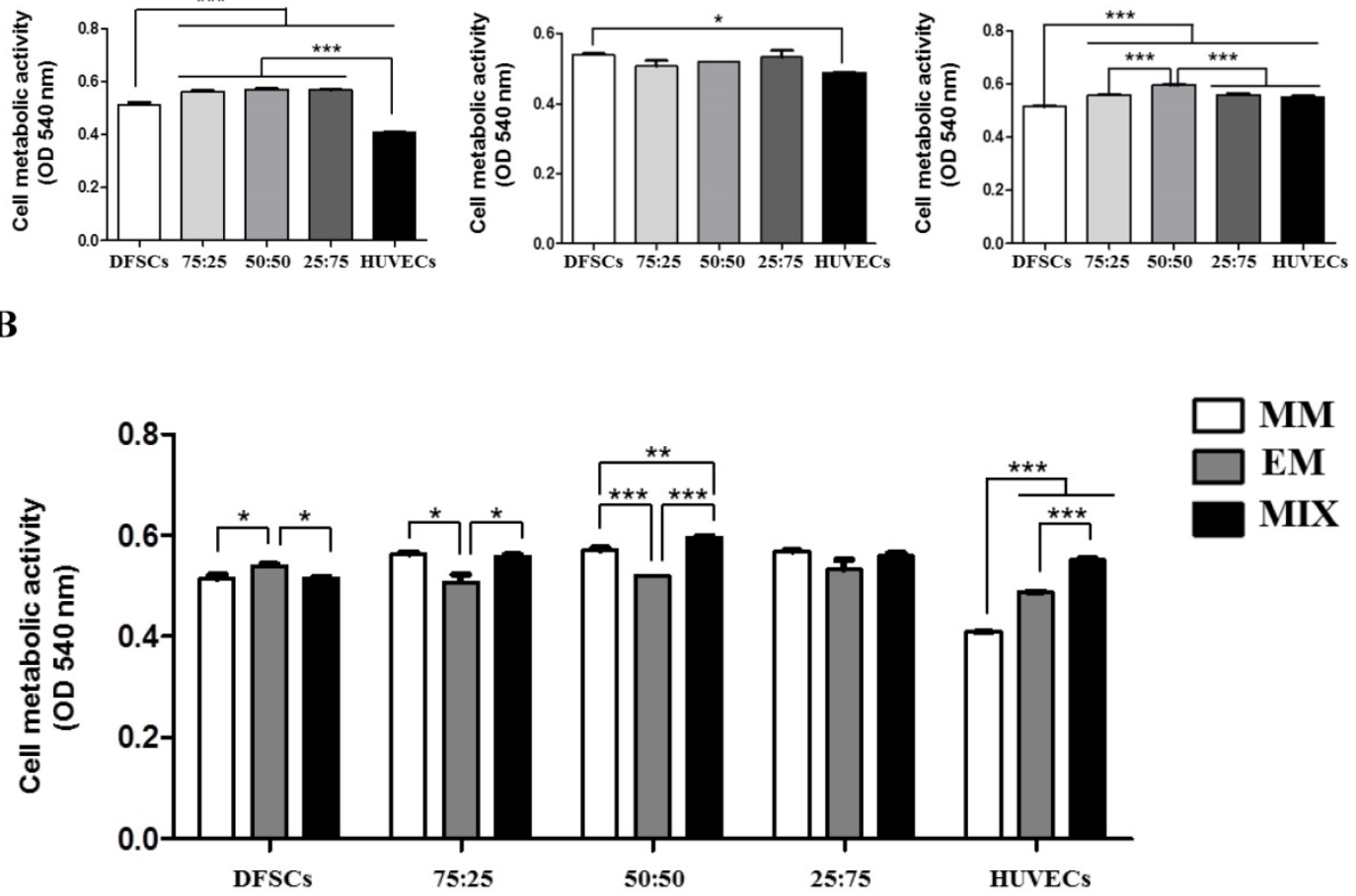

C
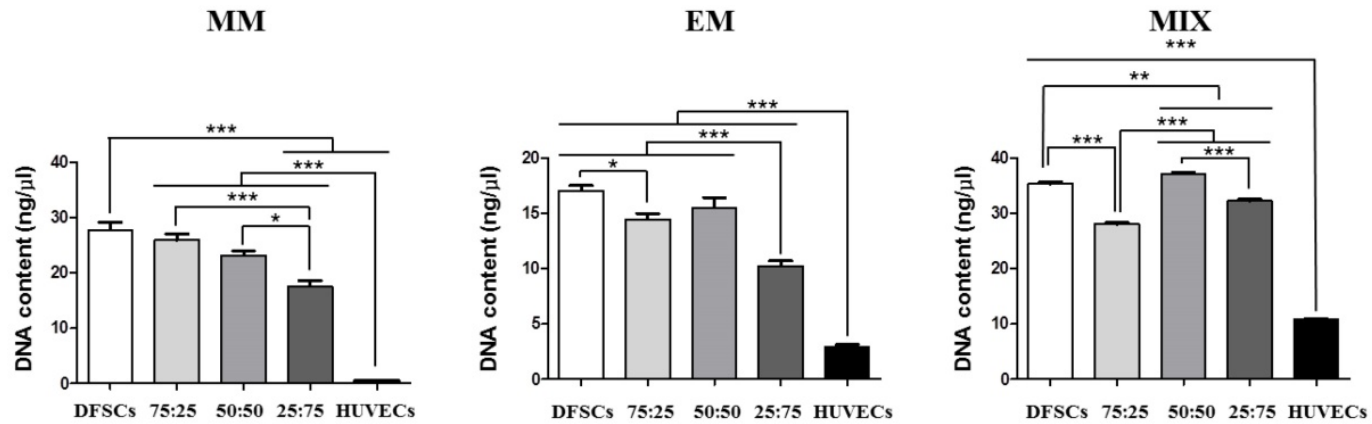

D

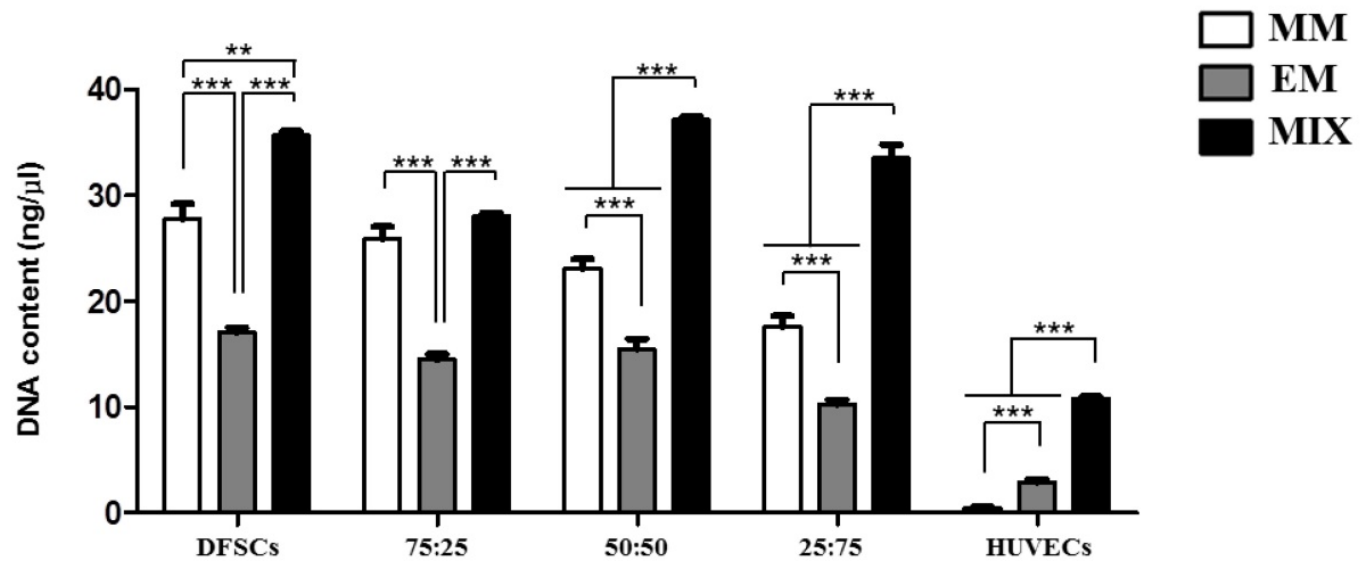

Figure 3. Cell metabolic activity and DNA content in co-cultures with different cell ratios (DFSC/HUVEC ratios of 100:0, 75:25, 50:50, 25:75, or 0:100) grown in different culture media (MM, EM, and MIX). A and B: Cell metabolic activity. C and D: DNA content. ${ }^{*}$ indicates $P<0.05$, ${ }^{* *}$ indicates $P<0.01$, and ${ }^{* *}$ indicates $P<0.001$. MM: MSCs growth medium; EM: endothelial cell growth medium; MIX: 1:1 mixture of MM and EM. 
A

B

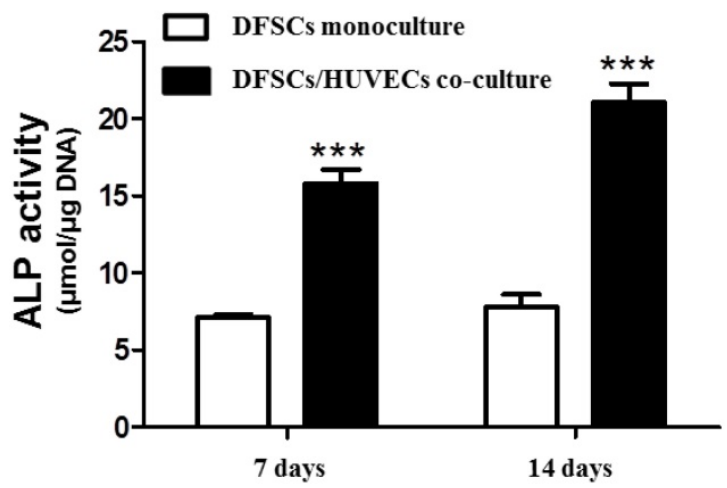

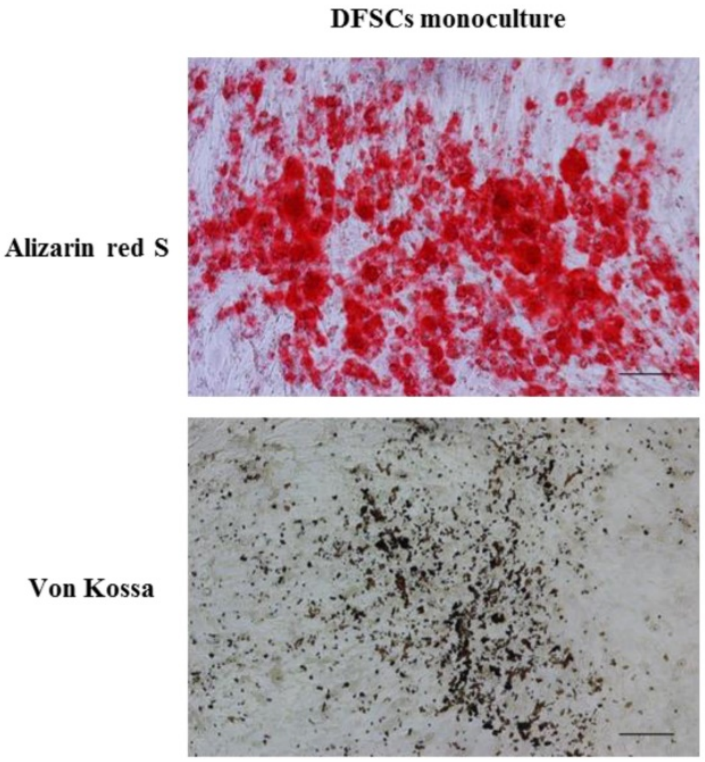

C
DFSCs/HUVECs co-culture
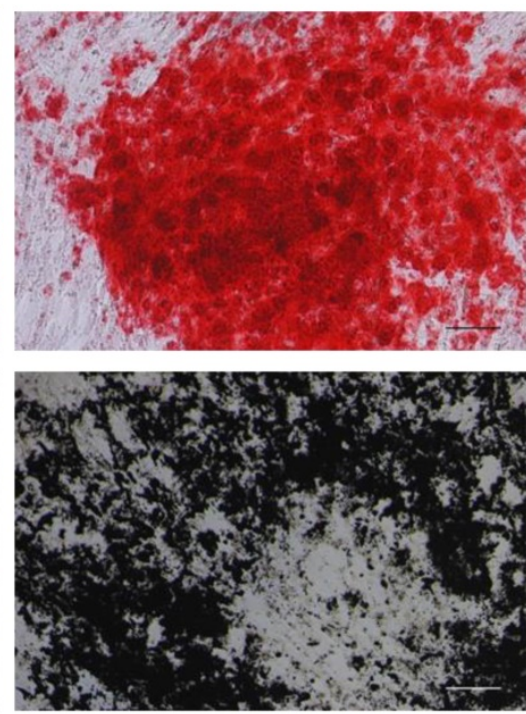

21 days

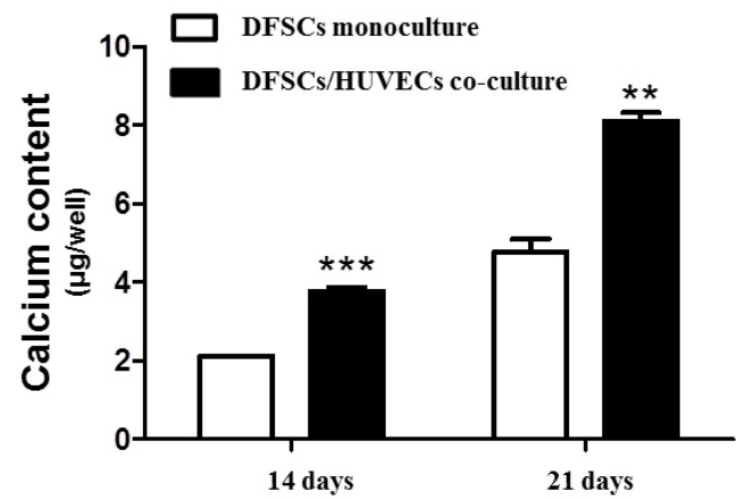

Figure 4. Osteoblastic phenotypes were enhanced in co-cultures with a DFSC/HUVEC ratio of 50:50. A: Bioactivity of ALP. B and C: Alizarin red- and von Kossa-positive mineralization and calcium content. Scale bar $=100 \mu \mathrm{m}$. ** indicates $\mathrm{P}<0.01$ and *** indicates $\mathrm{P}<0.001$.

VEGF protein levels were also significantly increased in 50:50 co-cultures when compared with those in DFSC monocultures at 7, 14 and 21 days of culture (Fig. 5B). Interestingly, we observed that VEGF and ANG1 mRNA expression, and VEGF protein levels increased over time in both 50:50 co-cultures and DFSC monocultures during the three-week experimental period. The time-dependent increase of angiogenic factors in both DFSC monocultures and DFSC/HUVEC co-cultures may be accompanied by osteoblastic maturation of both DFSC monocultures and 50:50 co-cultures [27]. In addition, 
A

VEGF

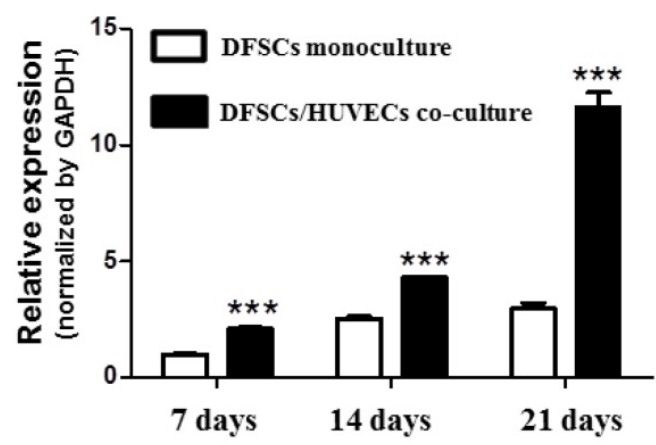

B

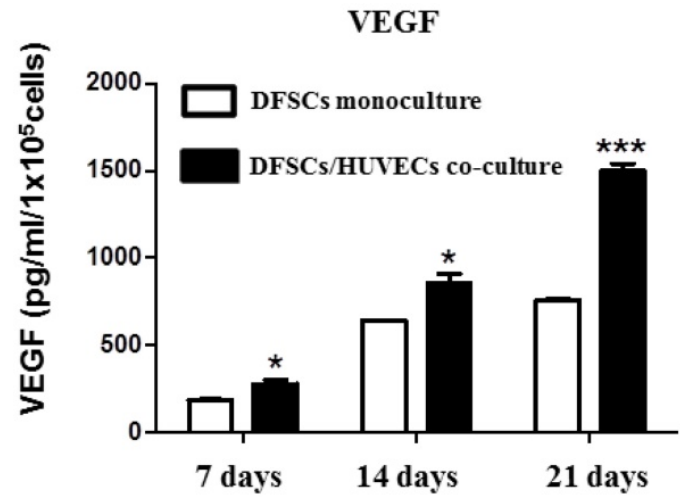

ANG1

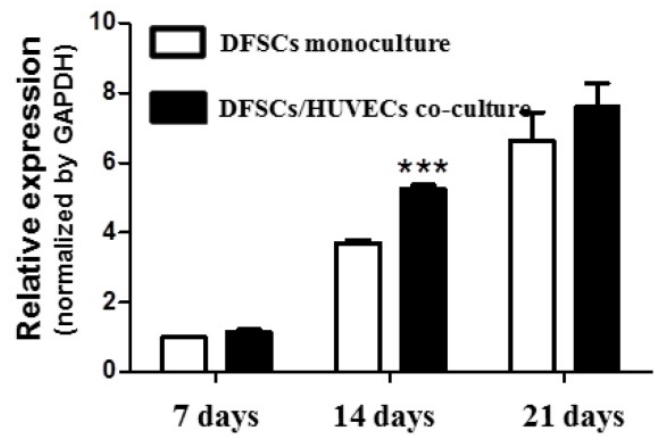

Figure 5. Angiogenic phenotypes in DFSC monocultures and co-cultures with a DFSC/HUVEC ratio of 50:50. A: qPCR analysis shows increased expression of angiogenic-related genes, VEGF and ANG1, in co-cultures with DFSC/HUVEC ratio of 50:50 B: VEGF protein levels showed a higher increase over 21 days in DFSC/HUVEC 50:50 co-cultures compared with those in DFSC monocultures.

our results also suggested that increased secretion of angiogenic factors in co-cultures of DFSCs and HUVECs may enhance osteoblastic maturation of DFSCs.

\section{Discussion}

The bone is a complex and highly vascularized tissue. Well-developed microvasculature and microcirculation systems are critical for the homeostasis and regeneration of bone. Physiological angiogenesis is a highly organized and tightly regulated process that is influenced by angiogenic and antiangiogenic factors, including several chemokines, depending on different tissue requirements. The disruption of oxygen supply/blood flow due to bone damage results in the formation of a hypoxic zone at the injury site that is thought to stimulate angiogenesis. During angiogenesis, endothelial cells get activated by angiogenic factors, which results in their proliferation, migration, and complete maturation to form new blood vessel networks. These networks support bone regeneration by delivering essential substances such as oxygen, nutrients, and regulatory cytokines. Aberrant angiogenesis might lead to pathogenesis in the bone tissue $[2,3,28]$.
Angiogenesis also influences the self-renewal and differentiation abilities of stem cells. Cells exhibiting stem cell-like properties have been isolated from different parts of the tooth, including the dental pulp, the apical papilla, and the dental follicle. Dental stem cells have similar characteristics to bone marrow-derived mesenchymal stem cells and can differentiate into osteoblasts and chondrocytes [29-33]. Dental stem cells can be harvested from donor tissue with relative ease (e.g. surgical extraction of an impacted third molar tooth) and, therefore, provide an attractive alternative to using bone marrow-derived stem cells for bone tissue engineering. In our study, dental follicle-derived cells expressed mesenchymal cell markers, such as CD44, CD73, CD90, and CD105, and were negative for the expression of hematopoietic stem cell markers, CD34 and CD45. These cells also successfully differentiated into mesenchymal cell lineages such as adipocytes and mature osteoblasts. Therefore, they can be classified as DFSCs.

Although angiogenic factors are important for the viability and differentiation of MSCs, their precise effects on the osteogenic differentiation of MSCs remain unclear [17-20]. HUVECs, which produce 
several types of angiogenic factors, are the most commonly used in vitro models to study the physiological and pathological processes of endothelial cells (e.g., angiogenesis). The effects of HUVECs on osteoblastic differentiation of dental stem cells remain unknown. This study used co-cultures of DFSCs and HUVECs to analyze the effects of angiogenic factors that are produced by HUVECs on the osteogenic maturation of dental stem cells [34-36].

CD31 is a cell adhesion molecule that is highly expressed at intercellular junctions of endothelial cells. It is a widely used marker for HUVECs. In our study, CD31 was expressed in HUVECs cultured in Medium 200 supplemented with LSGS. Therefore, Medium 200 supplemented with LSGS could be the optimal endothelial cell culture medium. Optimal co-culture conditions for MSCs and endothelial cells that ensure maximum viability of both cell-types have not yet been accurately determined [37-39]. We optimized DFSC/HUVEC co-culture conditions by measuring cell metabolic activity and DNA content to assess cell viability at different culture conditions. Cell viability was the highest in co-cultures with a DFSC/HUVEC ratio of 50:50 grown in a 1:1 mixed medium of MM and EM. Further analysis is needed to understand the effects of optimal co-culture conditions on the stem cell characteristics of DFSCs.

We found that osteoblastic and angiogenic phenotypes were more pronounced in co-cultures with a DFSC/HUVEC ratio of 50:50 compared with those in DFSC monocultures. We also observed a time-dependent increase in the expression of angiogenic phenotypes and VEGF protein levels in both 50:50 co-cultures and DFSC monocultures. These results suggested that the increase in osteogenic differentiation of DFSCs is accompanied by increasing levels of angiogenic factors in DFSCs, regardless of the presence of HUVECs. Therefore, the improved angiogenic activity seen in the presence of HUVECs may play a role in increased osteoblastic maturation of DFSCs in co-cultures.

The exact role that HUVECs play in osteoblastic differentiation of DFSCs through co-culturing HUVECs and DFSCs is not fully understood and needs further study. However, our study showed increased osteogenic and angiogenic phenotypes in DFSC/HUVEC co-cultures. In addition, our findings reinforce the idea that angiogenic factors, such as VEGF, that are secreted by HUVECs could play an important role in osteoblastic maturation of DFSCs.

\section{Acknowledgements}

This research was supported by Basic Science Research Program through the National Research Foundation of Korea (NRF) funded by the Ministry of
Education, Science and Technology (2015R1A5A 2008833, 2016R1D1A1B03932437). This study was also supported by a grant of the Korean Health Technology R\&D Project, Ministry of Health \& Welfare, Republic of Korea (HI13C1596, HI15C0607).

\section{Competing Interests}

The authors have declared that no competing interest exists.

\section{References}

1. Jung $\mathrm{Y}$, Song J, Shiozawa $\mathrm{Y}$, Wang J, Jung $\mathrm{Y}$, Song J, Shiozawa $\mathrm{Y}$, Wang J, Wang Z, Williams B, Havens A, Schneider A, Ge C, Franceschi RT, McCauley LK, Krebsbach PH, Taichman RS. Hematopoietic stem cells regulate mesenchymal stromal cell induction into osteoblasts thereby participating in the formation of the stem cell niche. Stem Cells. 2008;26(8):2042-2051.

2. Cui Q, Dighe AS, Irvine JN Jr. Combined angiogenic and osteogenic factor delivery for bone regenerative engineering. Curr Pharm Des. 2013;19(19):3374-3383.

3. Kang ML, Kim EA, Jeong SY, Im GI. Angiopoietin-2 Enhances Osteogenic Differentiation of Bone Marrow Stem Cells. J Cell Biochem. 2017;118(9):2896-2908.

4. Nancarrow-Lei R, Mafi P, Mafi R, Khan W. A Systemic Review of Adult Mesenchymal Stem Cell Sources and their Multilineage Differentiation Potential Relevant to Musculoskeletal Tissue Repair and Regeneration. Curr Stem Cell Res Ther. 2017;12(8):601-610.

5. Ansari S, Sarrion P, Hasani-Sadrabadi MM, Aghaloo T, Wu BM, Moshaverinia A. Regulation of the fate of dental-derived mesenchymal stem cells using engineered alginate-GelMA hydrogels. J Biomed Mater Res A. 2017;105(11):2957-2967.

6. Sharpe PT. Dental mesenchymal stem cells. Development. 2016;143(13):2273-80

7. Morsczeck C, Reck A, Beck HC. The hedgehog-signaling pathway is repressed during the osteogenic differentiation of dental follicle cells. Mol Cell Biochem. 2017:428(1-2):79-86.

8. Viale-Bouroncle S, Klingelhöffer C, Ettl T, Morsczeck C. The WNT inhibitor APCDD1 sustains the expression of $\beta$-catenin during the osteogenic differentiation of human dental follicle cells. Biochem Biophys Res Commun. 2015;457(3):314-317.

9. Rezai-Rad M, Bova JF, Orooji M, Pepping J, Qureshi A, Del Piero F, Hayes D, Yao S. Evaluation of bone regeneration potential of dental follicle stem cells for treatment of craniofacial defects. Cytotherapy. 2015;17(11):1572-1581.

10. Nie L, Yang X, Duan L, Huang E, Pengfei Z, Luo W, Zhang Y, Zeng X, Qiu Y, Cai T, Li C. The healing of alveolar bone defects with novel bio-implants composed of Ad-BMP9-transfected rDFCs and CHA scaffolds. Sci Rep. 2017;7(1):6373

11. Mori G, Ballini A, Carbone C, Oranger A, Brunetti G, Di Benedetto A, Rapone B, Cantore S, Di Comite M, Colucci S, Grano M, Grassi FR. Osteogenic differentiation of dental follicle stem cells. Int J Med Sci. 2012;9(6):480-487.

12. Subbiah R, Hwang MP, Van SY, Do SH, Park H, Lee K, Kim SH, Yun K, Park $\mathrm{K}$. Osteogenic/angiogenic dual growth factor delivery microcapsules for regeneration of vascularized bone tissue. Adv Healthc Mater. 2015;4(13):1982-1992.

13. Cui Q, Dighe AS, Irvine JN Jr. Combined angiogenic and osteogenic factor delivery for bone regenerative engineering. Curr Pharm Des. 2013;19(19):3374-3383.

14. Li B, Wang H, Qiu G, Su X, Wu Z. Synergistic Effects of Vascular Endothelial Growth Factor on Bone Morphogenetic Proteins Induced Bone Formation In Vivo: Influencing Factors and Future Research Directions. Biomed Res Int. 2016;2016:2869572

15. Veronesi F, Borsari V, Sartori M, Orciani M, Mattioli-Belmonte M, Fini M. The use of cell conditioned medium for musculoskeletal tissue regeneration. J Cell Physiol. 2018;233(6):4423-4442.

16. Zhang H, Kot A, Lay YE, Fierro FA, Chen H, Lane NE, Yao W. Acceleration of Fracture Healing by Overexpression of Basic Fibroblast Growth Factor in the Mesenchymal Stromal Cells. Stem Cells Transl Med. 2017;6(10):1880-1893.

17. Garcia P, Pieruschka A, Klein M, Tami A, Histing T, Holstein JH, Scheuer C, Pohlemann T, Menger MD. Temporal and spatial vascularization patterns of unions and nonunions: role of vascular endothelial growth factor and bone morphogenetic proteins. J Bone Joint Surg Am. 2012;94(1):49-58.

18. Zhang Y, Madhu V, Dighe AS, Irvine JN Jr, Cui Q. Osteogenic response of human adipose-derived stem cells to BMP-6, VEGF, and combined VEGF plus BMP-6 in vitro. Growth Factors. 2012;30(5):333-343.

19. Kyllönen L, Haimi S, Säkkinen J, Kuokkanen H, Mannerström B, Sándor GK, Miettinen S. Exogenously added BMP-6, BMP-7 and VEGF may not enhance the osteogenic differentiation of human adipose stem cells. Growth Factors. 2013;31(5):141-153

20. Sharma S, Sapkota D, Xue Y, Rajthala S, Yassin MA, Finne-Wistrand A, Mustafa K. Delivery of VEGFA in bone marrow stromal cells seeded in 
copolymer scaffold enhances angiogenesis, but is inadequate for osteogenesis as compared with the dual delivery of VEGFA and BMP2 in a subcutaneous mouse model. Stem Cell Res Ther. 2018;9(1):23.

21. Lee JH, Um S, Jang JH, Seo BM. Effects of VEGF and FGF-2 on proliferation and differentiation of human periodontal ligament stem cells. Cell Tissue Res. 2012;348(3):475-484.

22. Aksel H, Öztürk S, Serper A, Ulubayram K. VEGF/BMP-2 loaded three-dimensional model for enhanced angiogenic and odontogenic potential of dental pulp stem cells. Int Endod J. 2018;51(4):420-430.

23. Zhang $M$, Jiang $F$, Zhang $X$, Wang S, Jin $Y$, Zhang W, Jiang X. The Effects of Platelet-Derived Growth Factor-BB on Human Dental Pulp Stem Cells Mediated Dentin-Pulp Complex Regeneration. Stem Cells Transl Med. 2017;6(12):2126-2134.

24. Aksel H, Huang GT. Combined Effects of Vascular Endothelial Growth Factor and Bone Morphogenetic Protein 2 on Odonto/Osteogenic Differentiation of Human Dental Pulp Stem Cells In Vitro. J Endod. 2017;43(6):930-935.

25. Yoon DK, Park JS, Rho GJ, Lee HJ, Sung IY, Son JH, Park BW, Kang YH, Byun $\mathrm{SH}$, Hwang SC, Woo DK, Cho YC, Byun JH. The involvement of histone methylation in osteoblastic differentiation of human periosteum-derived cells cultured in vitro under hypoxic conditions. Cell Biochem Funct. 2017;35(7):441-452

26. Chung JE, Park JH, Yun JW, Kang YH, Park BW, Hwang SC, Cho YC, Sung IY, Woo DK, Byun JH. Cultured Human Periosteum-Derived Cells Can Differentiate into Osteoblasts in a Perioxisome Proliferator-Activated Receptor Gamma-Mediated Fashion via Bone Morphogenetic Protein signaling. Int J Med Sci. 2016;13(11):806-818

27. Mao L, Xia L, Chang J, Liu J, Jiang L, Wu C, Fang B. The synergistic effects of $\mathrm{Sr}$ and $\mathrm{Si}$ bioactive ions on osteogenesis, osteoclastogenesis and angiogenesis for osteoporotic bone regeneration. Acta Biomater. 2017;61:217-232.

28. Shi B, Andrukhov O, Berner S, Schedle A, Rausch-Fan X. The angiogenic behaviors of human umbilical vein endothelial cells (HUVEC) in co-culture with osteoblast-like cells (MG-63) on different titanium surfaces. Dent Mater. 2014;30(8):839-947.

29. Steimberg N, Angiero F, Farronato D, Berenzi A, Cossellu G, Ottonello A Kaigler D, Mazzoleni G. Advanced 3D Models Cultured to Investigate Mesenchymal Stromal Cells of the Human Dental Follicle. Tissue Eng Part C Methods. 2018;24(3):187-196.

30. Zhang J, Chen J. Bone Tissue Regeneration - Application of Mesenchymal Stem Cells and Cellular and Molecular Mechanisms. Curr Stem Cell Res Ther. 2017;12(5):357-364.

31. Fujii Y, Kawase-Koga Y, Hojo H, Yano F, Sato M, Chung UI, Ohba S, Chikazu D. Bone regeneration by human dental pulp stem cells using a helioxanthin derivative and cell-sheet technology. Stem Cell Res Ther. 2018;9(1):24.

32. Botelho J, Cavacas MA, Machado V, Mendes JJ. Dental stem cells: recent progresses in tissue engineering and regenerative medicine. Ann Med. 2017;49(8):644-651.

33. Pettersson LF, Kingham PJ, Wiberg M, Kelk P. In vitro Osteogenic Differentiation of Human Mesenchymal Stem Cells from Jawbone Compared with Dental Tissue. Tissue Engineering and Regenerative Medicine. 2017;14(6):763-774

34. Hernandez-Fernaud JR, Reid SE, Neilson LJ, Zanivan S. Quantitative mass spectrometry-based proteomics in angiogenesis. Proteomics Clin Appl. 2013;7(7-8):464-476.

35. Tan AW, Liau LL, Chua KH, Ahmad R, Akbar SA, Pingguan-Murphy B. Enhanced in vitro angiogenic behaviour of human umbilical vein endothelial cells on thermally oxidized TiO2 nanofibrous surfaces. Sci Rep. 2016;6:21828.

36. Ma J, Yang F, Both SK, Prins HJ, Helder MN, Pan J, Cui FZ, Jansen JA, van den Beucken JJ. In vitro and in vivo angiogenic capacity of BM-MSCs/HUVECs and AT-MSCs/HUVECs cocultures. Biofabrication. 2014;6(1):015005.

37. Ma J, van den Beucken JJ, Yang F, Both SK, Cui FZ, Pan J, Jansen JA. Coculture of osteoblasts and endothelial cells: optimization of culture medium and cell ratio. Tissue Eng Part C Methods. 2011;17(3):349-357.

38. Böhrnsen F, Schliephake H. Supportive angiogenic and osteogenic differentiation of mesenchymal stromal cells and endothelial cells in monolayer and co-cultures. Int J Oral Sci. 2016;8(4):223-230.

39. Takebe T, Sekine K, Enomura M, Koike H, Kimura M, Ogaeri T, Zhang RR, Ueno Y, Zheng YW, Koike N, Aoyama S, Adachi Y, Taniguchi H. Vascularized and functional human liver from an iPSC-derived organ bud transplant. Nature. 2013;499(7459):481-484. 